Relations industrielles

Industrial Relations

\title{
Compulsory Arbitration : Panacea or Millstone? By Harold S. Roberts, University of Hawaii, Industrial Relations Center, December 1965, 162 pages.
}

\section{Paul Guy}

Volume 22, numéro 1, 1967

URI : https://id.erudit.org/iderudit/027775ar

DOI : https://doi.org/10.7202/027775ar

Aller au sommaire du numéro

\section{Éditeur(s)}

Département des relations industrielles de l'Université Laval

\section{ISSN}

0034-379X (imprimé)

1703-8138 (numérique)

Découvrir la revue

Citer ce compte rendu

Guy, P. (1967). Compte rendu de [Compulsory Arbitration: Panacea or Millstone? By Harold S. Roberts, University of Hawaii, Industrial Relations

Center, December 1965, 162 pages.] Relations industrielles / Industrial Relations, 22(1), 142-143. https://doi.org/10.7202/027775ar

Tous droits réservés @ Département des relations industrielles de l'Université Laval, 1967
Ce document est protégé par la loi sur le droit d'auteur. L'utilisation des services d'Érudit (y compris la reproduction) est assujettie à sa politique d'utilisation que vous pouvez consulter en ligne.

https://apropos.erudit.org/fr/usagers/politique-dutilisation/ 
un intérêt particulièrement grand aux tomes deux et trois.

Inutile d'insister outre mesure en recommandant cet excellent ouvrage. Je suis certain qu'il saura s'imposer par lui-même. Je veux toutefois signaler l'importance pour les Canadiens français d'utiliser le volume traduit et adapté par monsieur Sylvain, car dans un marché aussi limité que le nôtre, il n'y a peut-être pos de place présentement pour plus d'un ourrage sur le sujet.

\section{Bertrand BELZILE}

The Suburban Society, par S.D. Clark, University of Toronto Press, Toronto Ontario, 1966,229 pages.

"There is no essential difference between new society of the suburbs and any other new society in terms of the kinds of forces which produced it $\mathbf{~ . ~}$

Tel est l'essentiel de la thèse que le sociologue torontois S.D. Clark soutient pour l'explication des sociétés banlieusardes. Cetto image semble aller à l'encontre de celle, plus communément admise, de Fourastié et de Riesman.

En effet, ce livre défie nos idées un peu stéréotypées au sujet des tendances de lo société moderne. Le professeur s'attache surtout ici aux grandes banlieues développées très rapidement qui, dit-il, ne démontrent pas les caractéristiques qu'on leur a attribuées jusqu'à maintenant. Pour lui, le banlieusard s'y est installé parce que le manque d'espace l'a forcé de quitter la ville. Ces centres, ayont eu un début difficile ont vite emprunté les caractéristiques urbaines. C'est là un des exemples les plus frappants des changements sociaux rapides de notre siècle.

$\mathrm{Ce}$ volume sera d'une grande utilité pour tous ceux qui s'intéressent à la ville, d̀ sa nature et à son développement.

\section{Poul GUY}

\section{The French Labor Courts: Judgment by} Peers, by William $\mathrm{H}$. McPherson and Frederic Meyers, Institute of Labor and Industrial Relations, University of Illinois, Urbana, 1966.104 pages.

Ce volume, à la fois onalytique et descriptif, vise à mettre en lumière le rôle et le fonctionnement des tribunaux du travail en France, tout en espérant que les outres pays, dont les Etats-Unis, bénéficient de cette expérience.

Après un bref aperçu historique, les auteurs nous présentent la structure d'organisation, le champ de la juridiction et les règles de procédure de ces tribunaux du travail.

Contrairement à ce que l'on rencontre dons la plupart des autres pays où il existe de semblables tribunaux, les juges ne sont pos nommés d'office par un organisme gouvernemental, mais plutôt élus en nombre égal parmi les représentants patronaux et syndicoux et, même parmi les travailleurs du rang. Leur mandat est d'une durée de six ans et, ils exercent leurs fonctions exclusivement au sein de la catégorie qu'ils représentent.

Le tribunal est habilité à rendre jugement sur l'ensemble des conflits de droit, qui peuvent survenir entre employeur et employé, soit à partir de la violation du contrat individuel de travail, de la législation, de la convention collective, ou tout simplement des usages et des coutumes dans l'industrie. Tout employeur ou tout employé peut recourir à ce tribunal, qu'il soit ou non assujetti d̀ une convention collective.

Une partie importante du présent ouvrage tente d'analyser et d'évaluer le fonctionnement actuel de ces tribuncux à partir de l'expérience quotidienne de la Cour de Paris. On y présente une étude des différentes causes, une oppréciation des divers documents disponibles, des observations personnelles sur les divers types de sessions et, de plus, une foule de renseignements recueillis auprès des juges, des représentants patronaux et syndicaux, et autres personnes en contact avec la Cour.

Cette brève appréciation de l'expérience française et des résultats positifs obtenus est sûrement de nature à favoriser l'évolution et l'amélioration du système nord-américain vers la création de véritables tribunaux du trovail répondant aux besoins de notre société. La parution de cet ouvrage ne peut que stimuler cet espoir et réjouir tous ceux qui luttent pour l'amélioration de notre système de relations industrielles.

\section{Gilles RICHARD}

Compulsory Arbitration: Panacea Or Millstone?, by Harold S. Roberts, University of Hawaii, Industrial Relations Center, December 1965, 162 poges.

L'auteur exprime ainsi le but de son livre: "What we have done here is to pre- 
sent four chapters which provide some background for the discussion of compulsory arbitration $\$$. II ne faut donc pas y chercher la défense d'une thèse quelconque, mais plutôt un ramassis des principaux problèmes ayant trait au sujet.

On retrouve d'abord des considérations théoriques et générales sur l'arbitrage: notes historiques, les différents types et leurs définitions que l'auteur évalue ensuite en les confrontant avec le réel. C'est ainsi qu'il relate les expériences des Etats-Unis et de quelques autres pays tels que I'Australie, la Grande-Bretagne, la France et la NouvelleZélande en matière d'arbitrage. Par souxi d'objectivité les arguments pour ou contre $c \in$ mode de règlement de conflit sont ensuite alignés.

Dans une autre partie, $M$. Roberts s'attache ou problème de l'intérêt public ou ce qu'il appelle les « Labor Disputes in Public Utilities ». Doit-on recourir à l'arbitrage obligatoire dans les conflits d'intérêt public? II suit la même démarche pour aborder cette question, i.e. il confronte les expériences avec les principales législations américaines qui s'y rapportent.

Le reste du volume se compose de commentaires de membres gouvernementoux, syndicaux ou patronaux s'affirmant pour ou contre l'arbitrage obligatoire. On s'en voudrait de ne pas souligner les oppendices relatant différents conflits industriels et l'excellente bibliographie au sujet de l'arbitrage et de l'expérience de plusieurs pays.

\section{Paul GUY}

Guide to the Quantitative Age, Readings from Fortune, Donald F. Mulvihill, Editeur, Holt, Rinehart and Winston, Inc., 1966, 233 pages.
Plusieurs phénomènes contemporains tels que l'automatisation et la conquête de l'espace nous soulignent éloquemment la place de plus en plus grande qu'occupent les modèles mathématiques et les ordinateurs dans notre civilisation. Comme le titre du présent volume I'indique, nous vivons à I' * âge quantitatif $\gg$. Le principal mérite de cette sélection consiste à nous offrir une lecture d'articles qui contribuent chacun à sa façon à nous faire mieux connaître cet aspect de notre civilisation.

Ces articles ne présenteront certes pas tous le même intérêt au lecteur ordinaire, mais tous me paraissent accessibles à ce dernier.

Le volume contient 14 articles qui ont parus dans la revue FORTUNE depuis 1949. L'énumération pure et simple de ces articles choisis, permettra au lecteur de bien saisir I'essentiel du contenu: « A Theory of Strategy », por John McDonald, (1949); * The War of Wits », par John MCDonald (1951); - The Business Game », par John McDonald et Franc Ricciardi, (1958); « The Science of Being Almost Certain 》, par George A. W. Boehm, (1964); \& The New Mathematics s, par George A.W. Boehm, (1958); - The New Uses of the Abstract $\#$, par George A.W. Boehm, (1958); "The Boundless Age of the Computer », par Gilbert Burck, (1964); Machines that Men Can Talk With », por John Pfeiffer, (1964); "Problems, Too, Have Problems », par John Pfeiffer, (1961); * Management Will Never Be the Same Again ", par Gilbert Burck, (1964); "Next, the Solid-State Vice President », par George A. W. Boehm, (1960); *Operations Research Is in Business *, par Herbert Solow, (1953); * Helping the Executive Make Up His Mind », par George A. W. Boehm, (1966) et a How to Cope with Information \$, par Francis Bello, (1960).

Bertrand BELZILE

\section{PUBLICATIONS RECENTES}

\section{GÉNÉRALITÉS}

* The Idea of Industrial Democracy in America, 1898-1915 by Milton Derber, Labor History, Vol. 7, No. 3, Fall 1966, pp. 259287

"The Social Sciences in the Age of Opulence », by Harry G. Johnson, The Canadian
Journal of Economics and Political Science, Vol. XXXII, No. 4, November 1966, pp. 423443.

"Proletarian Revolution and the Mass Strike $*$, by Charles F. Elliott, The Southwestern Social Science Quarterly, Vol. 47, No. 1, June 1966, pp. 44-51. 